\title{
A common basis for auditory sensory storage in perception and immediate memory
}

\author{
ROBERT G. CROWDER \\ Yale University, New Haven, Connecticut
}

\begin{abstract}
Thirty-two subjects participated in three experiments, one assessing auditory short-term memory for word lists with and without a verbal suffix and two assessing discrimination of synthetic vowels at either short or long interstimulus delays. The purpose was to find out whether the same kind of auditory memory supports both short-term memory and speech discrimination. There was a significant correlation between performance in the suffix and A-X speechdiscrimination experiments in those conditions likely to depend partly on echoic memory; however, there was no significant correlation between the tasks in conditions in which echoic memory was presumed to have been removed. The results provide a bridge between perception and memory procedures and support a theoretical model that was made to cover both domains.
\end{abstract}

The suffix effect is a decrement in recall of the last item in an immediate-memory list caused by an extra utterance (which does not have to be recalled) presented at the end of the list. Since the paper by Crowder and Morton (1969), one influential hypothesis for this phenomenon has been that a verbal suffix damages information that otherwise remains available, in sensory form, following auditory presentation. A survey of the research supporting that general position is available in Crowder, 1976 (chap. 3) and a recent, specific version of the hypothesis is in Crowder, 1978. The hypothesis is that speech sounds are represented, after they occur, on a two-dimensional, neutrally spatial grid that is organized by input channel and time of arrival. The entries on this grid are spectral descriptions of the speech sounds, similar to sound spectrograms. It is assumed (Crowder, 1978) that these representations are related to each other through the rules of recurrent lateral inhibition. From this, it follows that after a series of utterances on the same physical channel (i.e., the same voice in the same location), there will be lingering auditory information about the most recent arrival. This most recent item will be receiving lateral inhibition from only one direction, as opposed to the earlier items, which are inhibited from two directions. (The first few items in the series, including the very first, would not be prominent in the auditory system because of the sheer amount of time they have been undergoing

This research was supported by NSF Grant BNS 8005838 to R. Crowder and by NICHD Grant HD01994 and BRS Grant RR05596 to Haskins Laboratories. I acknowledge with pleasure the assistance of Virginia Walters for her help in all phases of this research, of Danny Kaye for warning me about the perils of correlating difference scores, and of Robert Sternberg and Bruno Repp for commenting on an earlier version of this paper. The author's complete address is: Department of Psychology, Yale University, New Haven, Connecticut 06520. mutual inhibition.) The freedom of the last utterance in a series from retroactive lateral inhibition is held responsible for the large recency effect observed in immediate memory tests with auditory presentation, but not with visual presentation (which does not activate the system under consideration here).

When a redundant suffix item is presented on the same channel as the memory list, just following the last to-be-remembered item, the latter loses its special status of being free from lateral inhibition from one direction, causing the suffix effect. The availability of this residual information about how the most recent item sounded is presumably used by the subject to supplement his regular categorical short-term memory for the items. This regular short-term memory is roughly the same whether the input modality is visual or auditory, but the auditory residual about the most recent item gives the latter modality the edge when the two are compared.

There are several recent pieces of research that may well force significant revision of this hypothesis for auditory memory (Ayres, Jonides, Reitman, Egan, \& Howard, 1979; Campbell \& Dodd, 1980; Spoehr \& Corin, 1978); however, the form of such a revision will likely leave intact the major assumptions about the suffix and modality effects and their common dependence on the same system (e.g., see Morton, Marcus, \& Ottley, 1981). It is probably fair to say that competing interpretations of the suffix effect have not yet been so thoroughly worked out as the one offered above. For example, those that propose specific hypotheses about how the suffix works often leave unexplained the modality effect (Spoehr \& Corin, 1978). Other competitors, such as the attentiongrouping suggestions of Kahneman and Henik (1981), seem to be dealing with a less molecular level of analysis than the explanation outlined above. When "grouping," for example, is used to explain some- 
thing, the next question is always, "What causes grouping?" Indeed, an explanation of grouping in the auditory system might well rely on principles of lateral inhibition!

In the speech-perception literature, it has been explicitly claimed for years that auditory memory plays an important role in speech discrimination experiments (Pisoni, 1973, 1975; Pisoni \& Tash, 1974; Fujisaki \& Kawashima, Note 1). The original idea here was that if phonetic category differences are not available to discriminate two similar speech tokens, they must be discriminated on the basis of their sounds. Since the sounds to be distinguished cannot ordinarily be presented simultaneously, this requires that the earlier item be remembered in sensory form until the later item, with which it is to be compared, has arrived.

The process assumption was that subjects try first to discriminate speech sounds on a phonetic basis and then go on to consult auditory memory only if the phonetic test fails. This "phonetic first" dual coding hypothesis has not fared very well empirically (Crowder, 1982; Pisoni, 1973; Repp, Healy, \& Crowder, 1979). These studies all varied the delay between two vowels being discriminated in the A-X (same/different) paradigm. It would be expected that, according to the dual-coding hypothesis, the withincategory discriminations would depend more on auditory short-term memory than the between-category discriminations. On the reasonable assumption that auditory memory decays faster than phonetic memory, then, the effect of a delay between the items being discriminated should be larger for the within- than for the between-category trials. Although Pisoni (1973, p. 258) reported this outcome verbally, there was a ceiling effect on between-category performance in discrimination hits (calling a true DIFFERENT trial "different"), and, with the d' performance measure, the decay slopes for within- and between-category trials were parallel. Crowder (1982) and Repp et al. (1979) obtained just the same result, parallel decay for within- and between-category discriminations along vowel continua, as a function of interitem delay.

However, the case for some role of auditory memory in vowel discrimination is a rather strong one, even if the phonetic-first, dual-code hypothesis is wrong: the fact that interstimulus delay causes deterioration in A-X vowel discrimination, by itself, is supportive of some role for auditory sensory memory in the task. This occurred reliably in the Pisoni (1973), Repp et al. (1979), and Crowder (1982) experiments. Furthermore, Pisoni (1975) showed that an interpolated vowel sound, placed immediately after target tokens in the ABX paradigm, significantly reduced performance compared with white-noise and tone controls. Repp et al. (1979) replicated this interference effect in the simpler, A-X task, by placing the interference sound midway between the two items being discriminated. Repp et al. suggested that this interference effect was the same disruption of auditory sensory memory that is observed in the suffix experiment.

The present experiments are aimed at strengthening the argument that the same auditory memory system serves both the suffix and vowel-discrimination tasks. The approach to be used relies on analysis of individual differences, rather than on experimental comparisons. The experimental work done in the past has produced three lines of evidence for a common auditory memory system in perception and short-term memory. The first point is the interference mentioned just above: in both the memory and perception experiments, an extra utterance seems to prevent the use of sound information for what just preceded the interfering item. In the suffix situation, it is the suffix that masks auditory memory for the last item on the list. In the vowel-discrimination setting, the masking vowel comes between the two sounds being distinguished in the A-X task (Repp et al., 1979).

The second point is that auditory memory in both situations seems to be subject to temporal decay. Crowder and Morton (1969) suggested that a life of approximately $2 \mathrm{sec}$ would be a plausible figure for the suffix experiment, and I have recently demonstrated (Crowder, 1982) that vowel-discrimination performance reaches asymptote when the A-X delay interval is approximately $3 \mathrm{sec}$.

The third point of similarity between the suffix and vowel-discrimination tasks is their common dependence on the phonetic class involved in the experiment. Pisoni (1973) first showed that the decay in A-X discrimination was much greater for stop consonants than for steady-state vowels. Crowder (1971) demonstrated that neither the modality effect nor the suffix effect occurs when the lists to be remembered contain items distinguished only by initial stop consonants. Crowder (1973) also demonstrated the same result with terminal stops. The fact that presumptive auditory-memory contributions come and go together as a function of phonetic class, in the two experimental settings, is consistent with the idea that they represent two manifestations of a common memory system.

Another strong point favoring this interpretation would be if individual subjects who showed a large auditory-memory capacity in the suffix task also showed a large auditory-memory capacity in the discrimination task. This outcome would cement the case for a common processing system in the two settings. But there are at least two circumstances that are discouraging from the very start of such an investigation of individual differences. One is that the auditory-memory contribution is numerically a small one compared with the effects of other variables in both experiments. The suffix effect is robust, but it 
is small in magnitude compared with the inventory of other established processes in immediate memory (encoding common to visual and auditory input, grouping, rehearsal, etc.). In vowel discrimination as well, the portion of performance that is sensitive to A-X delay, and therefore presumably the portion that shows auditory memory, is numerically very small (Crowder, 1982). So there is the risk that the performance components of interest are inherently swamped by other factors in any real experimental setting.

The second cautionary note is that the type of memory under consideration here may simply not differ much among people. If auditory memory in these settings is truly as sensory as as been claimed (Crowder, 1978), one might expect it to be relatively invariant and uninteresting from an individualdifferences standpoint. This is not to say that people are equivalent in their sensory capacities, of course. Indeed, it is hard to know how one could ever establish that people differ more in, say, working memory capacity than they do in visual acuity. However, in the context of tasks that are weighted more toward the complicated than toward the simple cognitive functions, it must be considered risky to be searching for individual differences in the simpler components. (An extreme example would be looking for individual differences based on visual acuity in the context of visually presented analogy problems.) For all these reasons, a negative outcome would not eliminate the case for a common memory system, but a positive outcome would be a striking victory for the theory.

\section{METHOD}

The subjects were taken through one suffix experiment and two vowel-discrimination experiments. The suffix effect has been well behaved in our laboratory for some time, and therefore there was little question how to conduct that part of the investigation. However, there are a number of possible discrimination paradigms, and it seemed undesirable to rely on only a single one of these. The traditional paradigm of choice in speech perception was for many years the so-called ABX paradigm, in which people hear three tokens of which the first two are different and they must decide whether the third is equal to one or the other of these first two. It has been claimed more recently (e.g., see Best, Morrongiello, \& Robson, 1981) that the ABX procedure systematically discounts auditory memory. This is because the second item in the ABX triad could serve to mask auditory storage of the first item until the third one arrives, and subjects may adopt the strategy of trying to compare the trace of the second item with the third. The A-X (same-different) procedure would seem better suited for showing auditory-memory effects because nothing comes between the two items being distinguished. By collecting data on the same stimuli and the same subjects in both $\mathrm{ABX}$ and $\mathrm{A}-\mathrm{X}$ procedures, it would be possible to compare the reliabilities and sensitivities of the two procedures directly. However, the main reason for using both ABX and A-X procedures was not to compare their sensitivities formally (which would require a much more extensive experiment to be definitive) but, rather, to maximize the chances of getting at least one discrimination task that could be associated with short-term memory.

\section{The Suffix Experiment}

Subjects and Materials. The subjects were 32 young adults of both sexes from our summer subject pool. Most, but not necessarily all, of them were college students during the academic year and were paid for their participation.

The stimuli were the nine digits, the nonsense syllable "ba," and a $1,000-\mathrm{Hz}$ tone. The verbal items were recorded by a male speaker and digitized on the Haskins Laboratories Pulse Code Modulation system, each in a 450 -msec time slot. These items were then accessible independently to other computer routines for automatically assembling the actual stimulus lists.

Design and Procedure. There were 20 trials in which nine-digit series were followed by the $1,000-\mathrm{Hz}$ tone and 20 in which the series were followed by the verbal suffix "ba."' On each trial, there was a 250-msec pause between each of the digits and between the last memory item and the redundant suffix or tone. Subjects were allowed $20 \mathrm{sec}$ for ordered written recall after each trial. Since there was no interest in looking at subtle properties of the suffix effect here, all subjects received the 20 control (tone) trials first and the 20 suffix trials second. (It will be seen below that not counterbalancing order of stimuli had no apparent effect on the suffix experiment as compared with numerous data sets in the literature in which these precautions were followed.) The instructions were standard in that they emphasized ordered recall and characterized the extra item (suffix or tone) as a cue telling people when to begin their recall attempt.

\section{The Discrimination Experiments}

The ABX and A-X experiments were conducted on the same 32 subjects as in the suffix experiment and directly after it. These two discrimination procedures were used in counterbalanced order, half the subjects starting with one and half with the other.

Stimuli. The stimulus items were all $300-\mathrm{msec}$ steady-state synthetic vowels produced on the Haskins Laboratories OVE IIIc synthesizer. There were eight different tokens ranging from /i/ to $/ \mathrm{I} /$ in approximately equal steps. The fundamental frequency for all tokens was brought from 90 to $100 \mathrm{~Hz}$ during the first $100 \mathrm{msec}$, remained at $100 \mathrm{~Hz}$ for the interior $100 \mathrm{msec}$, and then dropped to $85 \mathrm{~Hz}$ during the final $100 \mathrm{msec}$. The eight center frequencies of the first, second, and third formants, respectively, were: $F_{1}-269,287,304,320,339,356,372$, and $391 ; F_{2}-2,198$, $2,167,2,136,2,105,2,075,2,045,2,016$, and 1,$987 ; F_{3}-3,019$, $2,933,2,870,2,809,2,749,2,690,2,613$, and 2,557 . Overall amplitude for the vowels was constant over their duration. The materials were presented over loudspeakers at a comfortable level in a relatively quiet room.

Design. There were four blocks of speech-discrimination trials. For half of the subjects, the first two were $A B X$, and the second two were A-X; for the other half, this was reversed. The test stimulus (X) for either kind of discrimination trial was spaced at either a short $(500-\mathrm{msec})$ or a long $(3,000-\mathrm{msec})$ delay relative to the comparison stimulus (A in A-X or B in ABX tests). This was to affect the presence of auditory memory; details are given in the following sections. The design feature common to both discrimination procedures was that, for each task ( $A B X$ and $A-X)$, half the subjects had the short interval first and half had the short interval second. In other words, the scheduling of delay intervals across the four blocks of discrimination trials was either short-long-short-long or it was long-short-long-short. Again, there seemed no reason to avoid confounding the short-long order in the two paradigms because the project was aimed at individual differences rather than at point estimates for experimental effects.

The ABX task. On each ABX trial, there was first a 1,000-Hz tone, followed, after a 250 -msec delay, by the first of three vowel tokens relevant to that trial. Then, following a delay that was always set at $250 \mathrm{msec}$, the second of the three vowel sounds occurred. These first two vowels were always different tokens from the eight-item continuum. The delay between the second and the third of the items was the one that was varied to affect auditory 
memory decay; it was either 500 or 3,000 msec. There was then a 2,000-msec delay for the subject to record his response.

All possible one-step and two-step discriminations were tested in the ABX task. Consider the first two of the three vowels presented on a trial and call the eight vowels $1,2, \ldots, 8$. There are 14 one-step combinations (1-2, 2-1, 2-3, 3-2, 3-4, 4-3, etc.), and each of these has to be presented twice so that the correct answer is equally often the choice of " $A$ " and " $B$ " in the ABX triple (1-2-1, 1-2-2, 2-1-1, 2-1-2, 2-3-2, 2-3-3, etc.). Thus, there must be 28 different one-step trials. Analogously, there are 24 different two-step trials $(1-3-1,1-3-3,3-1-3,3-1-1,2-4-2,2-4-4$, etc.). The 52 possible $A B X$ trials were each presented once in the short-delay version and once in the long-delay version, for a total of $104 \mathrm{ABX}$ trials per subject. Within these constraints, the order of trials was random.

The ABX instructions stated that the first two vowels in a triple would always be different and that subjects should circle the number " 1 " or " 2 " on the answer sheet, depending on which of the first two vowels they thought matched the third.

The A-X task. The A-X task routine is, of course, simpler than the ABX because there are only two events on each trial instead of three. Following the tone, there was a 250 -msec pause, which was then followed by the first of the two vowels to be discriminated. After either a 500- or a 3,000-msec delay, the second vowel occurred, and the subject had $2,000 \mathrm{msec}$ to make his or her samedifferent response before the next trial started. The same 52 stimulus pairs used in ABX testing-28 one-step and 24 two-stepwere presented as the "different" trials in A-X. However, an additional 16 "same" pairs were added in which the two vowels were physically identical $(1-1,1-1,2-2,2-2$, etc.). This meant that a complete replication contained 68 trials, and two such replications were carried out, one for the short delay and one for the long delay. Instructions for the A-X procedure simply asked the subjects to circle the letters " $s$ " or " $d$ " on each trial, depending on whether or not the two vowels seemed to be "exactly the same sound."

\section{RESULTS}

The results will be presented in several sections. First, it will be established that each of the three separate experiments in this set produced reasonable results on its own, in terms of the existing literature. This is very much a precondition for examining individual differences among them. Second, the issue of formal reliability will be raised for the three data sets; this is another precondition, for if the measures are not reliable, there will be little use looking for individual differences. Finally, correlations among the different tasks will be considered.

\section{The Suffix Experiment}

Figure 1 shows the basic result of the suffix experiment. Every one of the subjects showed more errors in the suffix condition than in the control condition. For each condition there were 180 possible errors (20 trials $\times 9$ positions); the mean errors for the control and suffix conditions were, respectively, 42.75 and $69.47[\mathrm{t}(30)=8.19, \mathrm{p}<.0005]$. It is clear from the figure that the difference was located mainly toward the end of the list, most especially at the last serial position. In relation to the published literature, then, this was a thoroughly routine suffix experiment.

\section{The Discrimination Experiments}

Table 1 shows summary statistics from the ABX

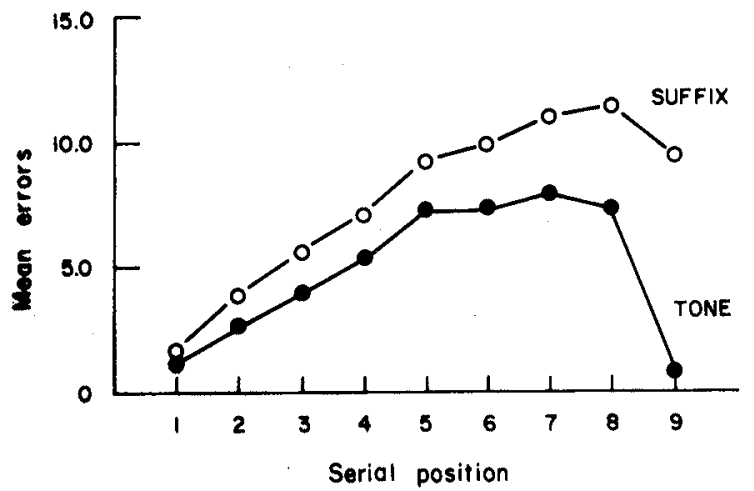

Figure 1. The relation between errors and input serial position in the suffix experiment.

and $\mathrm{A}-\mathrm{X}$ discrimination procedures. If these procedures are good tests of discrimination, it is reasonable to expect a large effect of step size, which in these experiments was set at either one or two. (In ABX tests of the continuum $1,2, \ldots, 8$, a one-step trial might present 1-2-2 and a two-step trial might present 1-3-3; in A-X tests, the corresponding trials could be 1-2 and 1-3.) The first section of Table 1 shows that indeed both procedures led to markedly fewer errors for the two-step than for the one-step trials. The ABX procedure, however, gave a smaller value of $t$ than the A-X procedure, 11.91 vs. 23.39.

The lower half of the table shows the data split according to the length of the delay interval, either the interval between $A$ and $X$ in the $A-X$ task or the interval between $\mathrm{B}$ and $\mathrm{X}$ in the $\mathrm{ABX}$ task. In both discrimination procedures, there was a higher error rate when this interval was long than when it was short; however, the difference was statistically significant only in the A-X task.

The data on discrimination as a function of delay were further examined using the tables of Kaplan, Macmillan, and Creelman (1978) for calculating d' from different discrimination paradigms. This analysis is shown in Table 2, in which the data are averaged over eight "supersubjects" of four individuals each, a grouping that was intended to minimize hit and false-alarm rates approaching zero and unity. The four subjects within a supersubject shared exactly the same counterbalancing condition: There were

Table 1

Speech Discrimination: Summary Statistics for Error Proportions

\begin{tabular}{llrr}
\hline & & \multicolumn{2}{c}{ Task } \\
\cline { 3 - 4 } Comparison & & ABX & \multicolumn{1}{c}{ A-X } \\
\hline \multirow{2}{*}{ Step Size } & One Step & .401 & .587 \\
& Two Step & .207 & .277 \\
& $\mathrm{t}(30)$ & 11.914 & 23.391 \\
\multirow{3}{*}{ Delay } & Short & .306 & .344 \\
& Long & .317 & .445 \\
& $\mathrm{t}(30)$ & .516 & 8.223 \\
\hline
\end{tabular}


Table 2

Sensitivity ( $\left(d^{\prime}\right)$ as a Function of Task and Delay

\begin{tabular}{ccc} 
& \multicolumn{2}{c}{ Task } \\
\cline { 2 - 3 } Interval & $\mathrm{ABX}$ & $\mathrm{A}-\mathrm{X}$ \\
\hline Short & 2.801 & 3.501 \\
Long & 2.247 & 2.910 \\
$\mathrm{t}(7)$ & 4.414 & 4.547 \\
\hline
\end{tabular}

two such control variables-whether $\mathrm{ABX}$ preceded A-X, or the other way around, and whether the short interstimulus intervals were tested first or second within each paradigm. Thus, there were four possible arrangements, and eight subjects, making up two supersubjects, received each. If Kaplan et al. (1978) are correct in asserting that these are fair measures of sensitivity across paradigms, then it may be concluded that the A-X task gives better discrimination than the ABX task $[t(7)=6.37, p<.0005]$. However, by this measure, the delay effect was reliable for both paradigms.

These analyses indicate that both discrimination experiments produced plausible results but that the A-X procedure might be more sensitive and therefore more useful for analyzing individual differences. The same conclusion comes from a formal analysis of reliability, which comes next.

\section{Reliability}

The best measure of the suffix effect, for purposes of ordinary experimentation, is probably some difference score, or ratio, representing how recency is changed on the last position across the suffix and control conditions. Although such measures have been useful for at least a decade of experimental work, they turn out to have limited reliability in in-

Table 3

Odd-Even Reliabilities

\begin{tabular}{|c|c|c|}
\hline \multirow[b]{2}{*}{ Measure } & \multicolumn{2}{|c|}{ Coefficient } \\
\hline & $\begin{array}{c}\text { Raw } \\
\text { Correlation }\end{array}$ & $\begin{array}{c}\text { Spearman-Brown } \\
\text { Corrected }\end{array}$ \\
\hline & \multicolumn{2}{|c|}{ Suffix Experiment } \\
\hline \multirow[t]{2}{*}{ Total Control Errors } & $\begin{array}{l}.937 \\
.927\end{array}$ & $\begin{array}{r}.967 \\
.962\end{array}$ \\
\hline & \multicolumn{2}{|c|}{ Discrimination Experiments } \\
\hline \multirow[t]{2}{*}{$\begin{array}{l}\text { Total ABX Errors } \\
\text { Total A-X Errors }\end{array}$} & $\begin{array}{l}.315 \\
.667\end{array}$ & $\begin{array}{l}.479 \\
.800\end{array}$ \\
\hline & \multicolumn{2}{|c|}{ A-X Discrimination } \\
\hline $\begin{array}{l}\text { Short Delay } \\
\text { Long Delay }\end{array}$ & $\begin{array}{l}.631 \\
.470\end{array}$ & $\begin{array}{l}.774 \\
.639\end{array}$ \\
\hline
\end{tabular}

Note-All correlations in the left column were reliable at $p<.01$ except total $A B X$ errors, which was not significantly different from zero $[t(30)=1.818]$. dividual differences analysis. Several such "pure" measures of the suffix effect, which show the group data of Figure 1 to good effect, gave odd-even reliabilities that were not significantly different from zero. The unreliability of difference scores is well documented (Cronbach \& Furby, 1970; Guilford, 1956).

The strategy followed here was to concentrate on measures from the suffix experiment that included, according to the theory, or did not include, according to the theory, a contribution from auditory memory. The control condition should contain this contribution, and the suffix condition should not. Table 3 shows the odd-even reliabilities of the total number of errors made in the control and suffix conditions, with and without the Spearman-Brown correction for attenuation. The odd-numbered trials were simply correlated with the even-numbered trials, over subjects, to produce these reliabilities. The SpearmanBrown correction enters the picture because there are only half as many observations in the two halves being correlated as there were on the original test. These reliabilities are highly reassuring and suggest that one could have designed this project with a shorter period of testing in the suffix experiment.

The odd-even reliabilities of the total errors made in the $\mathrm{ABX}$ and $\mathrm{A}-\mathrm{X}$ situations are also entered in Table 3, with and without the Spearman-Brown correction. (As in the suffix experiment, scores based on differences between the short and long delay interval-which should, theoretically, have been purer measures of auditory memory-were not at all reliable.) There is a clear basis for distinguishing the reliabilities of the ABX and A-X procedures here. The A-X procedure is more than twice as reliable, in the uncorrected data, as the ABX procedure. This may or may not be a general result: It is at least consistent with the stronger statistical evidence for step-size effects and for delay effects found in A-X compared with ABX testing. To repeat what was said earlier, the main purpose of this comparison was to come up with a suitable measure for comparing the suffix and discrimination experiments, not choosing the "best" discrimination task. Nonetheless, this result does suggest some caution for investigators choosing the ABX task, lest they be making it hard for themselves to demonstrate experimental effects in a sensitive way.

The third section of Table 3 shows odd-even reliabilities for the two main conditions of A-X discrimination, the short and long conditions. These ought to represent A-X discrimination with and without, respectively, the benefit of auditory memory, or, at least, there ought to be more auditory memory in the short than in the long condition. These reliabilities are satisfactory, although not as impressive as those that came from the suffix experiment. 


\section{The Relation Between Immediate Memory and Discrimination}

From the suffix experiment and from the A-X discrimination experiment, there are two scores for every subject, one in each experiment likely to include performance based on auditory memory and another likely not to include auditory memory. In the suffix experiment, the total performance in the control condition would be expected to include auditory memory but not performance in the suffix condition, because the suffix would have removed that component. In the A-X experiment, there should be an auditory component at the short interstimulus interval but not at the long interval, at which the auditory trace would have decayed.

Table 4 shows the relevant correlations. Notice, first, that there are large correlations between the two measures from both of the tasks. This indicates that there is a great deal of shared variance within either the suffix or discrimination experiments that, presumably, has nothing to do with auditory memory. In the upper right-hand quadrant of the table, the correlations are quite a bit lower, representing the relation between memory and speech discrimination. Of these four correlations, the only one that is different from zero, statistically, is the one that is presumed to contain the common component deriving from auditory memory. This reliable correlation of $.367(p<.025)$ is the major positive result of this set of experiments. In psychometric terms, it is not impressive in size, representing shared variance of about $13.5 \%$ between the two tasks. However, these psychometric criteria are not usually applied to data from straight experimental designs, for some reason. In terms of experimental work, rather, investigators typically celebrate when an a priori prediction specifying one of four conditions to exceed the other three comes out at better than the .025 level of confidence.

The highest of the other between-task correlations was $.278(p>.05)$. These other, nonsignificant, intertask correlations show that it was not just some general factor such as motivation or intelligence that produced the target relationship, for those factors would have led to relationships between all measures from the two experimental tasks. Rather, it must be

Table 4

Correlations Within and Between Memory and Discrimination Tasks

\begin{tabular}{lccc}
\hline & $\begin{array}{c}\text { Total } \\
\text { Control } \\
\text { Errors }\end{array}$ & $\begin{array}{c}\text { A-X } \\
\text { Short } \\
\text { Errors }\end{array}$ & $\begin{array}{c}\text { A-X } \\
\text { Long } \\
\text { Errors }\end{array}$ \\
\hline Total Suffix Errors & .853 & .278 & .262 \\
Total Control Errors & & .367 & .272 \\
A-X Short Errors & & & .731 \\
\hline
\end{tabular}

Note-t(30) values for $.278, .262, .367$, and .272 , respectively, are $1.59,1.49,2.16$, and 1.58 . counted a victory for the theory that the significant relationship occurred precisely where it was supposed to and nowhere else. (This is not to imply a much larger number of subjects would not push the three other intertask correlations to statistical reliability. There are other factors that might produce common variance in different laboratory tasks. The main point is that, within this particular study, it was only the expected correlation that was reliable.)

Furthermore, the obtained correlation of .367 is not quite as meager as it first seems. The square root of the reliability coefficient sets an upper limit on the variance that can be accounted for when the measure is correlated with anything external (validity). The square root of the odd-even reliability of total errors in the A-X short condition is .817 . The variance in the total errors from the control condition in the suffix experiment, accounted for by A-X short errors, was .135. Thus, the discrimination measure accounted for about $16.5 \%(.135 / .817)$ of the reliable variance in the suffix measure, which is not a disgrace considering the huge number of other components in both tasks.

\section{DISCUSSION}

One form of explanation in psychology is to relate the known properties of an experimental procedure to concepts that are more general than that specific procedure. It is often not terribly hard to offer a model for an experiment like the suffix experiment that accommodates its various properties neatly. Still, if the components of that model have no generality outside the suffix experiment, we are not satisfied that a true explanation has occurred. It is necessary to generalize components of the model to other settings in order to have a satisfying explanation.

There are several ways to establish generality of components across tasks. One is to show that the same experimental variables influence performance in the same way in each of two tasks. This much has been done in several areas. In short-term-memory experiments, for example, it has been shown that the suffix effect and also the visual-auditory modality effect disappear when the memory stimuli are distinguished only by stop consonants. Pisoni (1973) showed the vowel-stop consonant difference in speech discrimination. Likewise, interpolating an unrelated masking sound has a comparable interfering effect in both the memory and vowel-discrimination experiments. Thus, the two task settings respond quite similarly to certain experimental manipulations.

A second means of generalizing concepts across task settings is represented in this work-showing that individual differences in a theoretically specific component correlate reliably across the two tasks. People who show outstanding auditory memory in the immediate-memory control condition also show 
outstanding auditory memory in the A-X task with a short interstimulus interval. No single approach to this generalization of concepts is sufficient by itself, but when they operate in parallel, as they seem to here, one is justified in placing more weight on the explanatory power of the model in question. In this case, there seems to be even more reason, then, to take seriously the possibility that speech perception and short-term memory have some important information-processing processes in common.

\section{REFERENCE NOTE}

1. Fujisaki, H., \& Kawashima, T. On the modes and mechanisms of speech perception (Annual Report of the Engineering Research Institute). Tokyo: University of Tokyo, 1969.

\section{REFERENCES}

Ayres, T., Jonides, J., Reitman, J. S., Egan, J. C., \& Howard, D. A. Differing suffix effects for the same physical suffix. Journal of Experimental Psychology: Human Learning and Memory, 1979, 5, 315-321.

Best, C. T., Morrongiello, B., \& Robson, R. Perceptual equivalence of acoustic cues in speech and nonspeech perception. Perception \& Psychophysics, 1981, 29, 191-211.

CAmpbeld, A., \& DodD, B. Hearing by eye. Quarterly Journal of Experimental Psychology, 1980, 32, 85-89.

Cronbach, L. J., \& Furby, L. How we should measure "change" -or should we? Psychological Bulletin, 1970, 74, 68-80.

Crowder, R. G. The sounds of vowels and consonants in immediate memory. Journal of Verbal Learning and Verbal Behavior, 1971, 10, 587-596.

Crowder, R. G. Representation of speech sounds in precategorical acoustic storage. Journal of Experimental Psychology, 1973, 98, 14-24.
Crowder, R. G. Principles of learning and memory. Hillsdale, N.J: Erlbaum, 1976.

Crowden, R. G. Mechanisms of backward masking in the stimulus suffix effect. Psychological Review, 1978, 85, 502-504.

Crowder, R. G. Decay of auditory information in vowel discrimination. Journal of Experimental Psychology: Human Learning, Memory, and Cognition, 1982, 8, 153-162.

Crowden, R. G., \& Morton, J. Precategorical acoustic storage (PAS). Perception \& Psychophysics, 1969, 5, 365-373.

Guilford, J. P. Fundamental statistics in psychology and education. New York: McGraw-Hill, 1956.

Kahneman, D., \& Henik, A. Perceptual organization and attention. In M. Kubovy \& J. Pomerantz (Eds.), Perceptual organization. Hillsdale, N.J: Erlbaum, 1981.

Kaplan, H. L., Macmillan, N. A., \& Creelman, C. D. Tables of $d^{\prime}$ for variable-standard discrimination procedures. Behavioral Research Methods \& Instrumentation, 1978, 10, 796-813.

Morton, J., Marcus, S. M., \& Otrtexy, P. The acoustic correlate of "speechlike": A use of the suffix effect. Journal of Experimental Psychology: General, 1981, 110, 568-593.

Pisoni, D. B. Auditory and phonetic codes in the discrimination of consonants and vowels. Perception \& Psychophysics, 1973, 13, 253-260.

Pisoni, D. B. Auditory short-term memory and vowel perception. Memory \& Cognition, 1975, 3, 7-18.

Pisoni, D. B., \& TASH, J. Reaction times to comparisons within and across phonetic categories. Perception \& Psychophysics, $1974,15,285-290$.

Repp, B. H., Healy, A. F., \& Crowder, R. G. Categories and context in the perception of isolated, steady-state vowels. Journal of Experimental Psychology: Human Perception and Performance, 1979, 5, 129-145.

Spozhr, K. T., \& Corin, W. J. The stimulus suffix effect as a memory coding phenomenon. Memory \& Cognition, 1978, 6, 583-589.

(Manuscript received August 10, 1981; revision accepted for publication February 5, 1982.) 